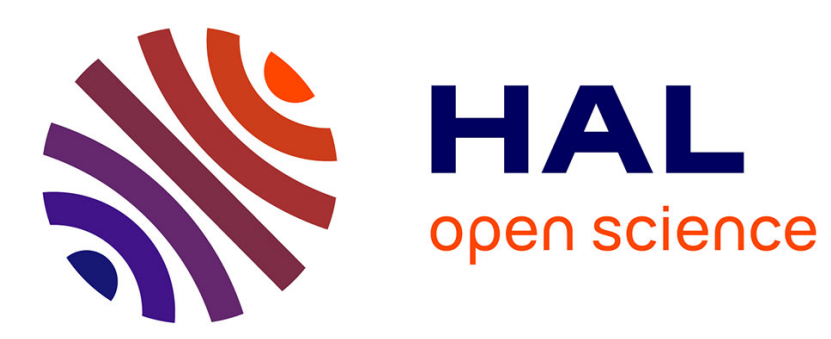

\title{
Kinematic analysis of a Meso-Scale Parallel robot for laser Phonomicrosurgery.
}

Sergio Lescano, Dimiter Zlatanov, Micky Rakotondrabe, Nicolas Andreff

\section{To cite this version:}

Sergio Lescano, Dimiter Zlatanov, Micky Rakotondrabe, Nicolas Andreff. Kinematic analysis of a Meso-Scale Parallel robot for laser Phonomicrosurgery.. Second Conference on Interdisciplinary Applications in Kinematics, IAK'13., Jan 2013, Peru. pp.1-8. hal-00878636

\section{HAL Id: hal-00878636 https://hal.science/hal-00878636}

Submitted on 30 Oct 2013

HAL is a multi-disciplinary open access archive for the deposit and dissemination of scientific research documents, whether they are published or not. The documents may come from teaching and research institutions in France or abroad, or from public or private research centers.
L'archive ouverte pluridisciplinaire HAL, est destinée au dépôt et à la diffusion de documents scientifiques de niveau recherche, publiés ou non, émanant des établissements d'enseignement et de recherche français ou étrangers, des laboratoires publics ou privés. 


\title{
Kinematic Analysis of a Meso-Scale Parallel Robot for Laser Phonomicrosurgery
}

\author{
Sergio LESCANO, Dimiter ZLATANOV, Micky RAKOTONDRABE, and \\ Nicolas ANDREFF
}

\begin{abstract}
The paper presents the kinematic model of a new meso-scale $\left(\sim 1 \mathrm{~cm}^{3}\right)$ parallel kinematic machine intended for laser phono-microsurgery of the vocal folds. The proposed mechanism uses the displacement generated by piezoelectric cantilever actuators manufactured via a Smart Composite Microstructure technique. The architecture, the geometry, and the position kinematics of the device, modeled as a spatial linkage, are discussed briefly. Then, the paper presents a velocity and singularity analysis and concludes that the new meso-scale parallel kinematic machine does not have singularities in the neighborhood of the reference configuration where it is required to operate.
\end{abstract}

Key words: Kinematic, Parallel Kinematic Machine, Screw Theory, Piezoelectric, Phonosurgery.

\section{Introduction}

Laser phonosurgery is a surgical procedure used to enhance the voice using a laser beam onto the vocal fold. This paper describes a meso-scale parallel kinematic machine (PKM) conceived with the aim to improve the quality of laser phono-surgery. This mechanism is placed at the end of a laryngoscope and orients a mirror used

Sergio LESCANO

Femto-ST Institute - Besançon - France. e-mail: sergio.lescano@femto-st.fr

Micky RAKOTONDRABE

Femto-ST Institute - Besançon - France. e-mail: mrakoton@femto-st.fr

Nicolas ANDREFF

Femto-ST Institute - Besançon - France. e-mail: nicolas.andreff@femto-st.fr

Dimiter ZLATANOV

University of Genoa - PMARlab Robotics Group - Genoa - Italy. e-mail: zlatanov@ dimec.unige.it 
to direct the laser beam onto the vocal fold. In order to account for all scale effects, to satisfy the constraints of the microworld, and to ensure the required accuracy, piezoelectric material $[1,2]$ combined with a smart composite microstructure (SCM) fabrication process $[8,9]$ are proposed to build this meso scale PKM.

In Section 2, the kinematic requirements of the meso-structure are described, the mechanism's architecture and geometry are proposed, and the position kinematics is discussed in brief. Section 3 is devoted to velocity and singularity analysis using screw theory. The main conclusions are drawn and possibilities for future work are outlined in Section 4.

\section{Kinematic Requirements and Proposed Structure}

\subsection{Kinematic Requirements}

Fig. 1 PKM, at the head of the laryngoscope, driving the laser beam direction onto the vocal fold.

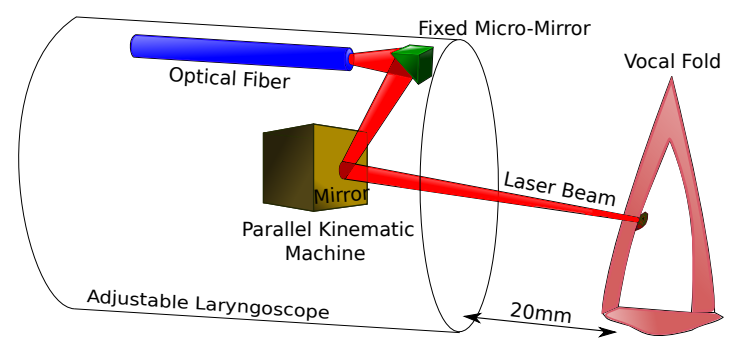

The PKM is located at the head of an adjustable laryngoscope. The laryngoscope's diameter is $20 \mathrm{~mm}$, and the space assigned for the robot is $10 \times 10 \times 10 \mathrm{~mm}^{3}$. The end of the laryngoscope is introduced into the cavity of the human larynx, at a distance of up to $20 \mathrm{~mm}$ from the vocal fold, as depicted in Fig. 1. To guide the direction of the laser beam onto the vocal fold, the PKM needs to have two rotational degrees of freedom (2 DOF). The standard average length of an adult vocal fold is between 17 and $20 \mathrm{~mm}$. With a working distance of up to $20 \mathrm{~mm}$, the PKM can scan the entire vocal fold if it can control the laser direction in a cone with a half-angle of $15^{\circ}$. The SCM manufacturing technique mentioned above, allows us to build the meso-scale robots as was shown by $[8,9]$, first in a plane and then folding and popping out to form the 3D structure.

Minimal bandwidth of $200 \mathrm{~Hz}$ and a scanning resolution better than $100 \mu \mathrm{m}$ are required to prevent overheating and damaging healthy tissue. Despite their small displacement, piezoelectric cantilever actuators have been chosen for their nanoresolution, work speed, and adaptability to the required scale [1, 2]. Piezoelectric cantilever actuators with dimensions of $10 \mathrm{~mm} \times 2 \mathrm{~mm}$ reach displacements of $100 \mu \mathrm{m}$. Piezoelectric cantilevers are arranged in parallel to not surpass the assigned dimensions. 


\subsection{Proposed Mechanism Architecture and Geometry}

The proposed PKM structure is depicted in Fig 2. The end-effector, or the moving platform, is used to orient a mirror, Fig 1.

The platform is connected to the base (attached to the laryngoscope) by a passive U-joint at $P$ (leg 3), which constrains it to only tilt and pan. We define the base reference frame Pxyz with coordinate unit vectors $\mathbf{i}, \mathbf{j}, \mathbf{k}$, and a rotating frame, fixed to the platform, with origin at $P$ and coordinate unit vectors $\mathbf{u}, \mathbf{v}, \mathbf{w}$, Fig. 2.

The 2-dof platform motion is actuated with two identical 6-dof RUS legs, legs 1 and 2. Piezoelectric actuation is used and modeled as the first R-joint of the each actuated leg, i.e., the piezoelectric cantilever assumed to be a rigid rod rotating about a fixed axis. The two active joints have a common axis, $A_{2} A_{1}$, with unit vector $\mathbf{k}_{1}=\sqrt{2} / 2 \mathbf{i}-\sqrt{2} / 2 \mathbf{j}$. (Point $A_{i}$ is the projection of the U-joint center $B_{i}$ on this axis.)

In each active leg, the axis of joint 2, the first joint of the passive $\mathrm{U}$-joint centered at $B_{i}, i=1,2$, is parallel to $A_{1} A_{2}$, while the center, $C_{i}$, of the spherical joint is along the common perpendicular of the U-joint axes.

At the reference configuration, when the angles of the U-joint at $P$ are $\theta_{1}=\theta_{2}=$ 0 , we have $\mathbf{c}_{1}=\overrightarrow{P C_{1}}$ parallel to $\mathbf{u}=\mathbf{i}$ and $\mathbf{c}_{2}=\overrightarrow{P C_{2}}$ parallel to $\mathbf{v}=\mathbf{j}$. Moreover, at the zero configuration, the distal links, $B_{i} C_{i}$, are parallel to the $z$ axis, while the proximal links, $A_{i} B_{i}$, are perpendicular to it and at equal angles with the $x$ and $y$ axes.

Each joint of the PKM is a compliant hinge and is made of a polyimide film which allows great flexibility without snapping [4], Fig 3 .

The dimensions of the PKM are given by $\left|A_{i} B_{i}\right|=20 l$ (corresponding to the piezoelectric-cantilever actuator), $\left|B_{i} C_{i}\right|=2 l,\left|P C_{i}\right|=l$, where $l=0.5 \mathrm{~mm}$. The distance between $P z$ and the common actuated-joint axis, $A_{1} A_{2}$, is $\left(20-\frac{\sqrt{2}}{2}\right) l$, while points $A_{1}$ and $A_{2}$ are at $\sqrt{2} l$ from each other. Therefore,

$$
\begin{aligned}
& \overrightarrow{P A_{1}}=-(10 \sqrt{2}-1) l \mathbf{i}-10 \sqrt{2} l \mathbf{j}-2 l \mathbf{k} \\
& \overrightarrow{P A_{2}}=-10 \sqrt{2} l \mathbf{i}-(10 \sqrt{2}-1) l \mathbf{j}-2 l \mathbf{k}
\end{aligned}
$$

\subsection{Position kinematics}

Given a feasible platform orientation, defined by the values of the passive-leg Ujoint angles, $\left(\theta_{1}, \theta_{2}\right)$, the mechanism configuration can be calculated by finding the intersection of a sphere centered at the point $C_{i}, i=1,2$, with radius $\left|B_{i} C_{i}\right|$ and the circle with center $A_{i}$, with radius $\left|A_{i} B_{i}\right|$, in a plane normal to line $A_{1} A_{2}$. In general, there will be four solutions of the inverse kinematics. Points $C_{i}$ are obtained as: 


$$
\begin{aligned}
& \overrightarrow{P C_{1}}=l \mathbf{u}=c_{2} l \mathbf{i}+s_{1} s_{2} l \mathbf{j}-c_{1} s_{2} l \mathbf{k} \\
& \overrightarrow{P C_{2}}=l \mathbf{v}=c_{1} l \mathbf{j}+s_{1} l \mathbf{k}
\end{aligned}
$$

where $c_{1}=\cos \theta_{1}, s_{1}=\sin \theta_{1}$. Indeed, the platform orientation is given by

$$
R\left(\theta_{1}, \theta_{2}\right)=R_{x}\left(\theta_{1}\right) R_{y}\left(\theta_{2}\right)=\left[\begin{array}{ccc}
c_{2} & 0 & s_{2} \\
s_{1} s_{2} & c_{1} & -s_{1} s_{2} \\
-c_{1} s_{2} & s_{1} & c_{1} c_{2}
\end{array}\right]
$$

We have $\overrightarrow{B_{j} C_{j}}=\overrightarrow{A_{j} C_{j}}-\overrightarrow{A_{j} B_{j}}$, and so

$$
\left|\overrightarrow{B_{j} C_{j}}\right|^{2}=4 l^{2}=\left|\overrightarrow{A_{j} C_{j}}\right|^{2}+400 l^{2}-2 \overrightarrow{A_{j} C_{j}} \cdot \overrightarrow{A_{j} B_{j}}
$$

where $\overrightarrow{A_{j} B_{j}}=10 l\left(\sqrt{2} c_{1}^{j} \mathbf{i}+\sqrt{2} c_{1}^{j} \mathbf{j}+2 s_{1}^{j} \mathbf{k}\right)$, and $c_{i}^{j}=\cos q_{i}^{j}, s_{i}^{j}=\sin q_{i}^{j}$. Using (1)(3) and (5) it is now possible to obtain a linear equation in terms of $c_{1}^{j}, s_{1}^{j}$

$$
F_{j} c_{1}^{j}+G_{j} s_{1}^{j}=H_{j}
$$

where $F_{j}, G_{j}$, and $H_{j}$ depend only on $\theta_{1}, \theta_{2}$, resulting in two solutions for $q_{1}^{1}$.

E.g. for $\theta_{1}=15^{\circ}, \theta_{2}=-15^{\circ}$ the solutions for $\left(q_{1}^{1}, q_{1}^{2}\right)$ are:

$$
\begin{array}{r}
\left(0.720102952001288^{\circ}, 0.742258398498497^{\circ}\right) \\
\left(0.720102952001288^{\circ}, 12.160623836841685^{\circ}\right) \\
\left(12.16311819616120381^{\circ}, 0.742258398498497^{\circ}\right) \\
\left(12.16311819616120381^{\circ}, 12.160623836841685^{\circ}\right)
\end{array}
$$

\section{Velocity and Singularity Analysis}

The velocity analysis is performed using screw theory, which has found a wide application, in particular in the the study of parallel robots. Instantaneous motion and forces acting on a rigid body can be represented by a twist and a wrench, respectively. With respect to a given reference frame, a twist (or a wrench) is given by a pair of (non-commensurate) physical vectors, $(\boldsymbol{\omega}, \mathbf{v})$, the body's angular velocity and the linear velocity at the origin (or $(\mathbf{f}, \mathbf{m})$, the resultant force and moment with respect to the origin). A normalized twists or wrenches has the same 3D geometrical representation, called a screw, defined by an axis and a pitch. (For a translation or a couple, there is no axis, but only a direction, and the pitch is said to be infinite.)

The reciprocal product, $\boldsymbol{\omega} \cdot \mathbf{m}+\mathbf{v} \cdot \mathbf{f}$, measures the power exerted by a wrench on a twist. Two screws, and any wrench-twist pair on them, are said to be reciprocal to each other if the product is zero. 
Fig. 2 Platform operated in parallel by two active legs and constrained by an unactuated U-joint leg.

Fig. 3 Realization of the passive U-joint.
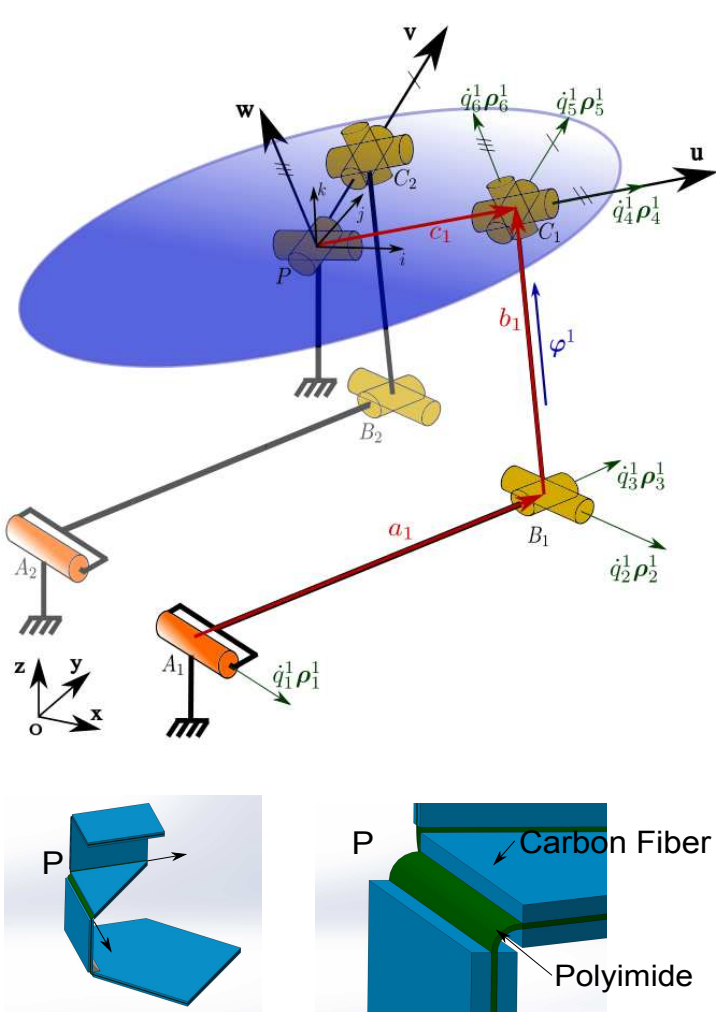

\subsection{Velocity kinematics}

On a PKM the instantaneous twist, $\boldsymbol{\xi}$, of the platform with respect to the ground can be expressed as a linear combination of the joint screws of leg $j$ [6]. Denoting by $\dot{q}_{i}^{j}$ and $\boldsymbol{\rho}_{i}^{j}$ the amplitude and the unit zero-pitch twist joint twist associated with the $i^{t h}$ joint of the $j^{t h}$ leg, we have:

$$
\begin{aligned}
& \boldsymbol{\xi}=\dot{q}_{1}^{1} \boldsymbol{\rho}_{1}^{1}+\dot{q}_{2}^{1} \boldsymbol{\rho}_{2}^{1}+\dot{q}_{3}^{1} \boldsymbol{\rho}_{3}^{1}+\dot{q}_{4}^{1} \boldsymbol{\rho}_{4}^{1}+\dot{q}_{5}^{1} \boldsymbol{\rho}_{5}^{1}+\dot{q}_{6}^{1} \boldsymbol{\rho}_{6}^{1} \\
& \boldsymbol{\xi}=\dot{q}_{1}^{2} \boldsymbol{\rho}_{1}^{2}+\dot{q}_{2}^{2} \boldsymbol{\rho}_{2}^{2}+\dot{q}_{3}^{2} \boldsymbol{\rho}_{3}^{2}+\dot{q}_{4}^{2} \boldsymbol{\rho}_{4}^{2}+\dot{q}_{5}^{2} \boldsymbol{\rho}_{5}^{2}+\dot{q}_{6}^{2} \boldsymbol{\rho}_{6}^{2} \\
& \boldsymbol{\xi}=\dot{\theta}_{1} \boldsymbol{\rho}_{1}^{3}+\dot{\theta}_{2} \boldsymbol{\rho}_{2}^{3}
\end{aligned}
$$

Since legs 1 and 2 have 6 dof they impose no constraint on the platform. Therefore the possible twists, $\boldsymbol{\xi}$, at every configuration, are given by $(8 \mathrm{c})$ as any linear combination of the passive leg's two revolute-joint unit twists, $\boldsymbol{\rho}_{1}^{3}$ and $\boldsymbol{\rho}_{2}^{3}$. When such a well-defined continuously-changing basis exists for the twist system of the 
platform, the velocity kinematics can be expressed with as few equations as the parallel mechanism's dof [12].

For this, one can first substitute (8c) into (8a) and (8b), and then eliminate the passive joint velocities using reciprocal screws obtaining a system of two equations relating the platform angular speeds $\dot{\theta}_{1}$ and $\dot{\theta}_{2}$ with the input velocities $\dot{q}_{1}^{1}$ and $\dot{q}_{1}^{2}$.

For each active leg, there is a unit pure force, $\boldsymbol{\varphi}^{j}, j=1,2$, reciprocal to all five passive-joint screws. Its axis passes through the centers of the leg's U- and S-joints.

(In a leg-singular configuration, where the center of the spherical joint is in the plane of the U-joint, there will be a whole pencil of concurrent planar forces with this property, and two, rather than one, reciprocal screws for that leg must be used to obtain the input-output velocity equations. We will assume that such leg postures do not occur during the operation of the mechanism.)

Taking the reciprocal product of $\boldsymbol{\varphi}^{1}$ with (8a) and of $\boldsymbol{\varphi}^{2}$ with (8b), we obtain:

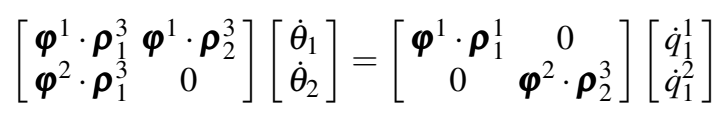

In (9), the term $\boldsymbol{\varphi}^{2} \cdot \boldsymbol{\rho}_{2}^{3}$ is zero, because the joint-2 axis of the passive leg always passes through point $C_{2}$. To calculate the other entries, we can use:

$$
\boldsymbol{\rho}_{1}^{3}=\left[\begin{array}{c}
\mathbf{i} \\
0
\end{array}\right], \quad \boldsymbol{\rho}_{2}^{3}=\left[\begin{array}{l}
\mathbf{v} \\
0
\end{array}\right], \quad \boldsymbol{\varphi}^{j}=\left[\begin{array}{c}
\frac{\mathbf{b}^{j}}{\left|\mathbf{b}^{j}\right|} \\
\mathbf{c}_{j} \times \frac{\mathbf{b}^{j}}{\left|\mathbf{b}^{j}\right|}
\end{array}\right], \quad \boldsymbol{\rho}_{1}^{j}=\left[\begin{array}{c}
\mathbf{k}_{1} \\
\overrightarrow{P A_{1}} \times \mathbf{k}_{1}
\end{array}\right], \quad j=1,2
$$

I Since $\overrightarrow{P A_{j}}=\mathbf{c}^{j}-\mathbf{b}^{j}-\mathbf{a}^{j}$ we have that

$$
\begin{aligned}
\boldsymbol{\varphi}^{j} \cdot \boldsymbol{\rho}_{1}^{j} & =\frac{\mathbf{b}^{j}}{\left|\mathbf{b}^{j}\right|} \cdot\left(\mathbf{c}^{j}-\mathbf{b}^{j}-\mathbf{a}^{j}\right) \times \mathbf{k}_{1}^{j}+\mathbf{k}_{1}^{j} \cdot\left(\mathbf{c}^{j} \times \frac{\mathbf{b}^{j}}{\left|\mathbf{b}^{j}\right|}\right) \\
& =\frac{\mathbf{b}^{j}}{\left|\mathbf{b}^{j}\right|} \cdot\left[\mathbf{k}_{1}^{j} \times \mathbf{a}^{j}\right] \quad j=1,2
\end{aligned}
$$

and so (9) can be written as:

$$
\left[\begin{array}{cc}
\frac{\mathbf{b}^{1}}{\left|\mathbf{b}^{1}\right|} \cdot \mathbf{k}_{1} \times \mathbf{a}^{1} & 0 \\
0 & \frac{\mathbf{b}^{2}}{\left|\mathbf{b}^{2}\right|} \cdot \mathbf{k}_{1} \times \mathbf{a}^{2}
\end{array}\right]\left[\begin{array}{c}
\dot{q}_{1}^{1} \\
\dot{q}_{1}^{2}
\end{array}\right]=\left[\begin{array}{cc}
\mathbf{c}^{1} \times \frac{\mathbf{b}^{1}}{\left|\mathbf{b}^{1}\right|} \cdot \mathbf{i} & \mathbf{c}^{1} \times \frac{\mathbf{b}^{1}}{\left|\mathbf{b}^{\top}\right|} \cdot \mathbf{v} \\
\mathbf{c}^{2} \times \frac{\mathbf{b}^{2}}{\left|\mathbf{b}^{2}\right|} \cdot \mathbf{i} & 0
\end{array}\right]\left[\begin{array}{c}
\dot{\theta}_{1} \\
\dot{\theta}_{2}
\end{array}\right]
$$

which can be abbreviated as $B \dot{\mathbf{q}}=D \dot{\boldsymbol{\theta}}$, where $B$ is a diagonal matrix.

\subsection{Singularity Analysis}

Gosselin and Angeles suggested a classification of singularities for parallel manipulators into three main groups associated with the singularity of square matrices such 
as $D$ and $B$ above [3]. Later Zlatanov gave a more comprehensive study of the singularities of closed-loop mechanisms including configurations where the matrices $D$ and $B$ are not square $[10,11]$. This can occur in the case mentioned above of either leg 1 or leg 2 being in a singular posture with the center of the S-joint lying in the plane of the U-joint, in which case a redundant passive mobility singularity is present. However, if we assume that such leg postures are outside of the range of operation of the mechanism, the singularities of the mechanism are the configurations where either $B$ or $D$ is singular, leading to a degeneration of the inverse or the direct instantaneous kinematics, respectively.

In (9) or (12), matrix $B$ is singular when one or both elements of its diagonal becomes zero, signifying that the actuated joint axis intersects one of the reciprocal force axes. In this case, if $D$ is nonsingular, we have a singularity of type (IO, RI), where the end-effector loses freedoms and the input velocities can be nonzero with a stationary platform.

This cannot happen in a neighborhood of the reference configuration where $\mathbf{q}_{1}^{j}=$ 0 , matrix $B$ is equal to $\operatorname{diag}\left(\left|\mathbf{a}_{1}^{1}\right|,\left|\mathbf{a}_{2}^{1}\right|\right)$ far from having a zero determinant.

To find a singularity of the matrix $D$, the axis of either $\varphi^{1}$ or $\varphi^{2}$ must lie in the plane of the passive-leg U-joint. In this case, the platforms freedoms cannot be controlled. It will be able to move with actuators locked and the actuated velocities are not independent and cannot be chosen arbitrarily, a singularity of types RO and II. Here again, with a good choice of geometric parameters, the PKM is safe in a neighborhood of the position where $\mathbf{q}_{1}^{j}=0$ with

$$
D=\left[\begin{array}{cc}
0 & -\left|\mathbf{c}^{1}\right| \\
\left|\mathbf{c}^{2}\right| & 0
\end{array}\right]
$$

Since the PKM workspace is limited to the angles required to scan entire vocal fold $\left( \pm 15^{\circ}\right)$, both $B$ and $D$ are expected nonsingular, in this reduced workspace.

\section{Conclusion}

This paper presents a meso-scale PKM dedicated to the laser phonomicrosurgery of vocal folds. A kinematic analysis is performed. Reciprocal screws are used to find the two matrices that describe the mechanism's velocity behavior. All possible cases of singularities are analyzed. The limitation of the workspace and the proposed mechanism geometry avoid each type of singularity.

In the future, owing to the microfabrication limits and the robot structure compliance, a study of the directional variation of the screws will be taken into account using interval analysis [5]. Moreover, due to the low displacement generated by piezoelectric cantilever actuators, a different configuration in a position near-singular for the matrix $D$ could be exploited [7] to generate large platform rotations with small displacements of the actuators, being near the point of singularity without reaching it to preserve controllability. 


\section{Acknowledgments}

This work has been supported by the European Union Seventh Framework Programme [FP7-ICT-2011-7]. Project $\mu$ RALP (www.microralp.eu).

\section{References}

[1] Ballas, R.: Piezoelectric Multilayer Beam Bending Actuators: Static and Dynamic Behavior and Aspects of Sensor Integration. Microtechnology and Mems. Springer (2007)

[2] Clévy, C., Rakotondrabe, M., Chaillet, N.: Signal Measurement and Estimation Techniques for Micro and Nanotechnology. Springer (2011)

[3] Gosselin, C., Angeles, J.: Singularity analysis of closed-loop kinematic chains. Robotics and Automation, IEEE Transactions on 6(3), 281 -290 (1990)

[4] Lobontiu, N.: Compliant Mechanisms: Design of Flexure Hinges. Mechanical engineering. CRC Press (2003)

[5] Merlet, J.P.: Interval analysis and reliability in robotics. International Journal of Reliability and Safety 3 (2009)

[6] Mohamed, M.G., Duffy, J.: A Direct Determination of the Instantaneous Kinematics of Fully Parallel Robot Manipulators. Journal of Mechanisms Transmissions and Automation in Design 107 (1985)

[7] Voglewede, P.A., Ebert-Uphoff, I.: Two Viewpoints on the Unconstrained Motion of Parallel Manipulators at or Near Singular Configurations. In: International Conference on Robotics and Automation, pp. 503-510 (2002)

[8] Wood, R., Avadhanula, S., Menon, M., Fearing, R.: Microrobotics using composite materials: the micromechanical flying insect thorax. In: Robotics and Automation. IEEE International Conference on, vol. 2, pp. 1842-1849 (2003)

[9] Wood, R.J., Avadhanula, S., Sahai, R., Steltz, E., Fearing, R.S.: Microrobot design using fiber reinforced composites. Journal of Mechanical Design 130(5), $052,304+(2008)$

[10] Zlatanov, D., Benhabib, B., Fenton, R.: Analysis of the instantaneous kinematics and singular configurations of hybrid-chain manipulators. Proceedings of the ASME 23rd Biennial Mechanisms Conference 72, 467 - 476 (1994)

[11] Zlatanov, D., Fenton, R., Benhabib, B.: Identification and classification of the singular configurations of mechanisms. Mechanism and Machine Theory 33(6), 743 - 760 (1998)

[12] Zoppi, M., Zlatanov, D., Molfino, R.: On the velocity analysis of interconnected chains mechanisms. Mechanism and Machine Theory 41(11), 1346 1358 (2006) 